Nuclear Physics B323 (1989) 253-266

North-Holland, Amsterdam

\title{
AN INCLUSIVE AND EXCLUSIVE ALGORITHM FOR QED EVOLUTION*
}

\author{
Giovanni BONVICINI \\ University of Michigan, Ann Arbor, MI 48109, USA \\ Luca TRENTADUE \\ Dipartimento de Fisica, Università di Parma, INFN, Gruppo Collegato di Parma, \\ 43100 Parma, Italy
}

Received 8 February 1989

\begin{abstract}
A novel algorithm to calculate radiative corrections to $\mathrm{e}^{+} \mathrm{e}^{-}$annihilation which is both inclusive and exclusive is proposed. The method uses the structure functions formalism and is based on the factorization of mass singularities in the QED perturbation expansion. The general approach is presented together with its ability to reproduce the infrared and collinear singularity structure of QED as well as the all-order multiple photon radiation. Some numerical results are given.
\end{abstract}

\section{Introduction}

In the last years a powerful way to calculate QED radiative corrections (RC) in $\mathrm{e}^{+} \mathrm{e}^{-}$experiments [1] has been brought to the attention of the scientific community. Given the need of very high precision predictions at LEP/SLC, such an approach has already been used to calculate RC to inclusive quantities [2-5]. In this work we tackle the problem of a complete description of the evolution of a given QED process by using the structure function formalism. We build an algorithm which generates an arbitrary number of photons along with their individual 4-momenta. and which is suitable for a general application of RC to experimental data.

This algorithm is intended for both inclusive and exclusive processes. By inclusive, we mean those reactions where the final state is only partially reconstructed or computed; in an exclusive reaction the entire observable final state is reproduced. Historically, inclusive calculations have always achieved high precision for a small subset of observables [6,7]. At LEP/SLC, for example, analytic formulae describe precisely the inclusive shape of the $Z^{0}$. The entire class of weak corrections can be

* Work supported by the Department of Energy, contract DE-AC02-84ER01112; and by the Istituto Nazionale de Fisica Nucleare. 
dealt with analytically too, the reason being that no extra weak bosons are radiated. However, RC to data are in most cases an integral over a multidimensional space. There are three degrees of freedom for each observed particle. Extra dimensions and irregular integration boundaries are added by detector acceptances, resolutions, efficiencies and imposed cuts. As is well-known [8], a Monte Carlo procedure is the only way to perform such complex integrals. Finite-order perturbation methods, which are not precise in the most densely populated regions of the phase space, are strictly exclusive. This originates from allowing only a limited number of photons, which in turn forces the introduction of an unphysical cutoff. However, they were found suitable for Monte Carlo event generation [9] and have been far more extensively used in applications to experiments than inclusive methods.

Here we present a method that both has aspects of a correct infrared behaviour and which allows generation of exclusive final states. The former aspect, together with its main consequence of a correct inclusion of multiple all-order photon radiation, is now believed to be a natural ingredient of any radiative correction scheme. It has also the feature of keeping track explicitly of the transverse degrees of freedom. In this we differ from other QED structure function Monte Carlos, which have a fixed number of photons and only a part of the correct multiphoton 4-momentum [10-12].

This paper is organized as follows: In sect. 2 we discuss the poissonian properties of evolution equations in longitudinal phase space. We limit ourselves to the evolution of the initial state in $\mathrm{e}^{+} \mathrm{e}^{-}$annihilation. In sect. 3 we implement the transverse degrees of freedom and add modifications as needed to push the precision of the algorithm beyond $1 \%$. In sect. 4 we present some results and conclusions.

\section{The poissonian properties of evolution equations}

The analogy with QCD Drell-Yan type processes carried out in refs. [1-5] is based on a Lipatov-Altarelli-Parisi evolution equation for electron or positron states which in the non-singlet channel, i.e. for the evolution of a single valence electron or positron state without any sea contribution, is

$$
D(x, s)=\delta(1-x)+\frac{\alpha}{2 \pi} \int_{m^{2}}^{s} \frac{\mathrm{d} s^{\prime}}{s^{\prime}} \int_{x}^{1} \frac{\mathrm{d} z}{z} P(z) D\left(\frac{x}{z}, s^{\prime}\right) .
$$

$P(z)$ is the regularized $\mathrm{e} \rightarrow \mathrm{e}+\gamma$ vertex given by

$$
P(z)=\frac{1+z^{2}}{1-z}-\delta(1-z) \int_{0}^{1} \mathrm{~d} t \frac{1+t^{2}}{1-t} .
$$

The second (virtual) term is zero everywhere but at $z=1$. The density $D(x, s)$ 
represents the probability of finding inside a parent electron (positron) of mass $m$, at the scale $s$, an electron (positron) with a fraction $x$ of a given kinematic variable. According to the majority of QCD-type applications, $\mathrm{e}^{+}$and $\mathrm{e}^{-}$are evolved independently, and we define, for now,

$$
x=p_{\mathrm{L}} / E=1-k \cos \theta .
$$

Here $k$ is the fractional energy of the emitted photon in units of the beam energy $E$, $p_{\mathrm{L}}$ is the longitudinal momentum and $\theta$ is the angle between the emitted photon and the radiating leg. The cross section is derived from a convolution integral over the electron and positron legs

$$
\sigma(s)=\int \mathrm{d} x_{1} \mathrm{~d} x_{2} \sigma_{0}\left(s^{\prime}\right) D\left(x_{1}, s\right) D\left(x_{2}, s\right)
$$

and $s^{\prime}=x_{1} x_{2} s$. Iterated and truncated to first order, eq. (1) is

$$
D(x, s)=B(0) \delta(1-x)+B(1) P(x) .
$$

The $B(n)$ are the probability to radiate $n$ photons above a physical cutoff (see sect. 4) during the evolution, and in the unitary approximation they play the role of branching ratios. In the following, we will implicitly assume the kernels multiplying the $B(n)$ to be unitary, so that all the information on the branching probability will be contained in the $B(n)$, which we will now determine. Iterated to third order, eq. (1) has the form

$$
\begin{aligned}
D(x, s)=\delta(1-x)+ & \frac{\boldsymbol{\alpha}}{2 \pi}\left[\int \frac{\mathrm{d} s^{\prime}}{s^{\prime}} P(x)+\frac{\alpha}{2 \pi}\left[\int \frac{\mathrm{d} s^{\prime \prime}}{s^{\prime \prime}} \int \frac{\mathrm{d} z}{z} P\left(\frac{x}{z}\right) P(z)\right.\right. \\
& \left.+\frac{\alpha}{2 \pi}\left[\int \frac{\mathrm{d} s^{\prime \prime \prime}}{s^{\prime \prime \prime}} \int \frac{\mathrm{d} z}{z} \int \frac{\mathrm{d} y}{y} P\left(\frac{x}{y z}\right) P(z) P(y)+\cdots\right]\right] .
\end{aligned}
$$

Upon integrating in $x$ each term, above and below the dimensionless cutoff $x_{0}$, one extracts the coefficients $B(n)$. We drop non-dominant terms which correspond to the sea electrons, and find

$$
\begin{aligned}
& B(0)=1-\bar{n}+\frac{\bar{n}^{2}}{2}-\frac{\bar{n}^{3}}{6}+\cdots=\mathrm{e}^{-\bar{n}}, \\
& B(1)=\bar{n} B(0), \\
& B(2)=\frac{1}{2} \bar{n}^{2} B(0), \quad \text { etc. }
\end{aligned}
$$


with

$$
\bar{n}=(\alpha / \pi) L(-l-3 / 4), \quad L=\log \left(s / m^{2}\right), \quad l=\log \left(x_{0}\right)
$$

The probability distribution is poissonian $[6,13]$ with an average number of photons depending logarithmically on the cutoff. By forcing the emission probability to be binomial, zero or one emitted photon, and imposing the condition that both probabilities be positive, we find

$$
B(0)=1-\bar{n}, \quad B(1)=\bar{n}, \quad 0<\bar{n}<1
$$

This is the well-known limit on the cutoff that affects truncated expansion Monte Carlos [9], or the equivalent result given by eq. (5). Finally, the substitution $L \rightarrow L-1$ in eq. (8) correctly normalizes the emission probability [14] and reproduces, by direct inspection of the matrix elements, all the dominant terms up to order $\alpha^{2}$, and the $l$-dominant terms [2]. A calculus theorem can be used to transform the double emission term in eq. (6) (the proof for $n$ photons is automatic) into an explicit probability density

$$
\int_{x /\left(1-x_{0}\right)}^{1-x_{0}} \frac{\mathrm{d} z}{z} P(z) P\left(\frac{x}{z}\right)=\frac{\mathrm{d}}{\mathrm{d} x} \int_{\Omega} \mathrm{d} z^{\prime} \mathrm{d} z^{\prime \prime} P\left(z^{\prime}\right) P\left(z^{\prime \prime}\right)
$$

where the integration area $\Omega$ is defined by

$$
z^{\prime}, z^{\prime \prime}<1-x_{0}, \quad z z^{\prime}>x
$$

From eqs. (7) and (10) it appears that the iterative solution of eq. (1) is an infinite sum of terms with poissonian normalization, and each term is given by an iterated convolution of an elementary kernel. The details of the proof for eq. (10) can be found in the appendix.

Having generated a poissonian photon multiplicity, the algorithm proceeds to generate the longitudinal variable for each photon according to the kernel in eq. (2). In the case of two photons, the steps are:

(1) Generate $z_{1}, P(z)$ as defined in eq. (2), and $0<z<1-x_{0}$.

(2) Repeat the first step for $z_{2}$.

(3) Let $x=z_{1} z_{2}, k_{1}=1-z_{1}$ and $k_{2}=z_{1}\left(1-z_{2}\right)$.

The second photon is allowed by the evolution to have an energy $z_{1} x_{0}<k_{1}<z_{1}$, part of which is below the cutoff (fig. 1). As the phase space for the second photon is reduced, the cutoff is moved inward to maintain a poissonian photon multiplicity. The probability of radiating a photon $k_{1}$ and a second photon $k_{2}$ below the cutoff 

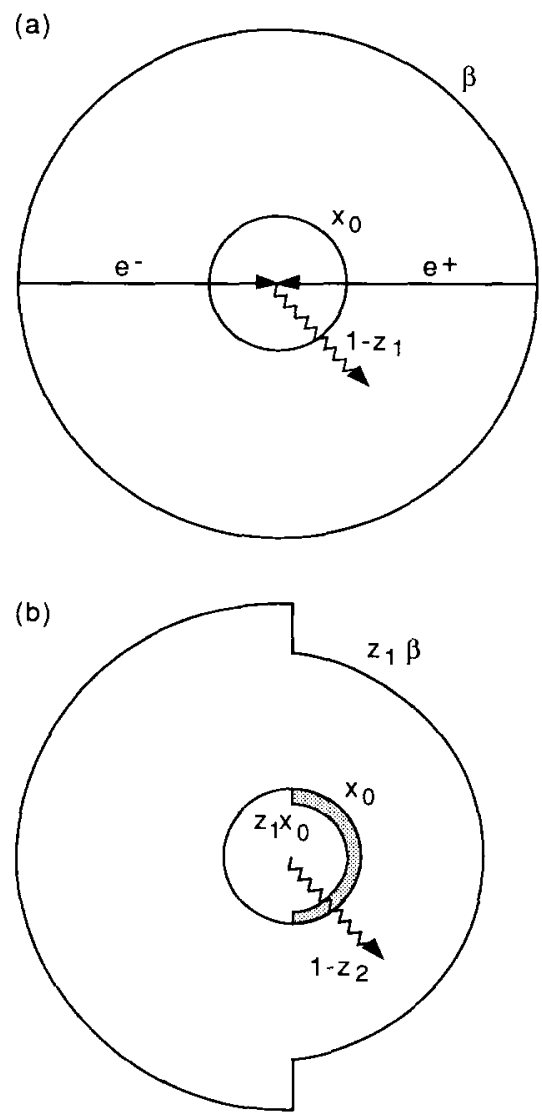

Fig. 1. Evolution of the cutoff in the approximation that the electron and positron evolve independently: (a) phase space allowed to the first photon; (b) phase space allowed to the second photon.

is given by the integral of the $P(z)$ over the shaded area in fig. $1 \mathrm{~b}$. The result is:

$$
\left(\frac{\alpha L}{2 \pi}\right)^{2} \int_{1-x_{0}}^{1-z_{1} x_{0}} \frac{2 \mathrm{~d} z_{2}}{1-z_{2}} \frac{1+z_{1}^{2}}{1-z_{1}}=\left(\frac{\alpha L}{\pi}\right)^{2} \frac{1}{2} \log z_{1} \frac{1+z_{1}^{2}}{1-z_{1}}
$$

which has to be compared with those non-poissonian $\alpha^{2} L^{2}$ terms [3] where one real photon plus one soft-virtual photon are radiated. The two expressions are identical and the virtual-virtual (no real photons) and real-real (two real photons) terms are easily proven to be factorizable [3] in the $\alpha^{2} L^{2}$ terms. We conclude that our variable cutoff method achieves factorization of the $L$-dominant terms [2]. 


\section{Inclusion of transverse degrees of freedom}

Eq. (1) can be generalized [15] to take also into account transverse degrees of freedom. In QED, by keeping the masses and by using the correct kinematics, the evolution equation becomes

$$
\frac{\partial D\left(x, p_{\mathrm{T}}, s^{\prime}\right)}{\partial s^{\prime}}=\frac{\alpha}{2 \pi} \frac{1}{s^{\prime}-m^{2}} \int_{x}^{1} \frac{\mathrm{d} z}{z} P(z) \int \frac{\mathrm{d}^{2} q_{\mathrm{T}}}{\pi} \delta_{k}^{*} D\left(\frac{x}{z}, p_{\mathrm{T}}-\frac{x}{z} q_{\mathrm{T}}, s^{\prime}\right)
$$

The $D\left(x, p_{\mathrm{T}}, s\right)$ represents the probability to find inside the parent $\mathrm{e}^{-}\left(\mathrm{e}^{+}\right)$an $\mathrm{e}^{-}\left(\mathrm{e}^{+}\right)$with a fraction $x$ of a longitudinal variable and a 2 -vector transverse variable $p_{\mathrm{T}}$ with respect to the parent direction. We define, for now, the $p_{\mathrm{T}}^{2}$ as

$$
p_{\mathrm{T}}^{2}=E^{2} k^{2} \sin ^{2} \theta
$$

so that the variables $x$ and $p_{\mathrm{T}}$ are independent components of a 3-vector. The $\delta_{k}^{*}$ function guarantees a correct kinematic splitting at the vertex. In the space-like (initial state) case [16], the argument of $\delta_{k}^{*}$ at the $i$ th step of the evolution is

$$
(1-z) M_{i+1}^{2}-z(1-z) M_{i}^{2}-q_{\mathrm{T}}^{2}
$$

where $M_{i+1}^{2}<0$ is the virtualness of the inner leg, $M_{i}^{2}<0$ is the virtualness of the outer leg, and $M_{1}^{2}=-m^{2}$ (fig. 2). We repeat the steps of sect. 2. To $O(\alpha)$ we find [16]:

$$
\begin{aligned}
D\left(x, p_{\mathrm{T}}, s\right)= & B(0) \delta(1-x) \delta^{2}\left(p_{\mathrm{T}}\right) \\
& +B(1) P(x)\left[p_{\mathrm{T}}^{2}-(1-x)^{2} M_{1}^{2}\right]+\mathrm{O}\left(p_{\mathrm{T}}^{2} / E_{\gamma}^{2}\right),
\end{aligned}
$$

and for the total cross section we have

$$
\sigma(s)=\int \mathrm{d} x_{1} \mathrm{~d} x_{2} \mathrm{~d}^{2} p_{\mathrm{T} 1} \mathrm{~d}^{2} p_{\mathrm{T} 2} \sigma_{0}\left(s^{\prime}\right) D\left(x_{1}, p_{\mathrm{T} 1}, s\right) D\left(x_{2}, p_{\mathrm{T} 2}, s\right),
$$

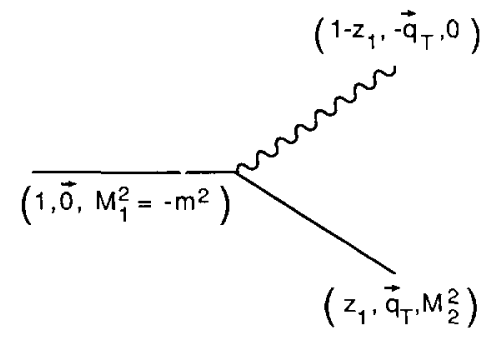

Fig. 2. Four-momentum conservation at the elementary vertex. The quantities are defined in the text. 
where

$$
s^{\prime}=2\left(\sqrt{x_{1}^{2} E^{2}+p_{\mathrm{T} 1}^{2}} \sqrt{x_{2}^{2} E^{2}+p_{\mathrm{T} 2}^{2}}+x_{1} x_{2} E^{2}-p_{\mathrm{T} 1} \cdot p_{\mathrm{T} 2}\right)
$$

In sect. 2 we discussed how to generate the photon multiplicity and longitudinal variables, and according to eqs. (15) and (16) the following complete set of formulae hold at the $i$ th step of the iteration [see also Alg. (11)]:

$$
\begin{aligned}
x_{i} & =\prod_{j=1}^{i} z_{j}, & k_{\mathrm{L} i} & =\prod_{j=1}^{i-1} z_{j}\left(1-z_{i}\right), \\
p_{\mathrm{T} i} & =q_{\mathrm{T} i}+x_{i} p_{\mathrm{T} i-1}, & k_{\mathrm{T} i} & =-q_{\mathrm{T} i}+\left(1-x_{i}\right) p_{\mathrm{T} i-1} .
\end{aligned}
$$

The $k_{\mathrm{L}}, k_{\mathrm{T}}$ are the $x, p_{\mathrm{T}}$ variables for the photon. Fig. 3 shows that in eq. (19) the evolution variable $q_{\mathrm{T}}$ is the photon transverse momentum with respect to the instantaneous direction of the leg and that eq. (19) simply conserves the 3-momentum at each step of the evolution. The evolution of the energy of the leg is trivially derived from the zero mass of the photon. At the end of the evolution of the initial state, the electrons and positrons which are off mass shell are used to calculate the value of the annihilation cross section by evaluating the Born term at the reduced center-of-mass energy [eq. (18)]. This completes the description of the basic algorithm for the QED evolution.

The basic structure-function approach was designed to carry up to leading logarithmic factorization of the evolution. Without losing any of the advantages of the main algorithm described above, we have introduced two modifications to achieve the second-order next-to-leading result and agreement with a first-order calculation in the very hard region $\left(p_{\mathrm{T}}^{2} \approx s\right)$, as needed for a powerful and high precision (less than $1 \%$ ) prediction.

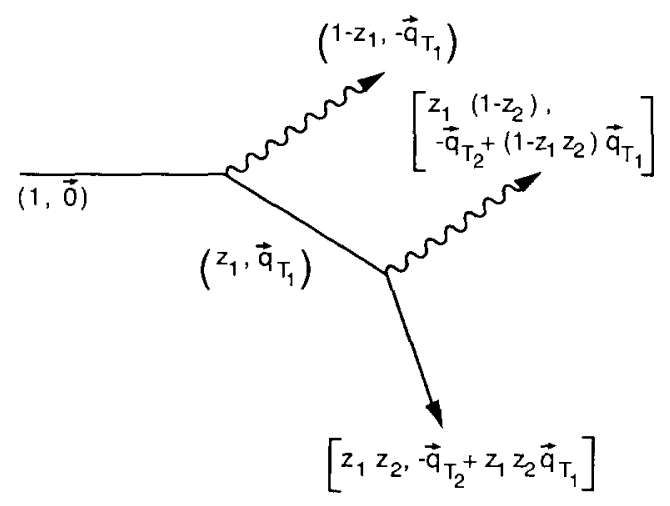

Fig. 3. Evolution of the leg. The quantities are defined in the text. 
We compare with an exact first-order calculation [17] (which we have calculated using REDUCE [18]) and include further less dominant terms to achieve the $O(\alpha)$ matrix element up to $\mathrm{O}\left(\mathrm{m}^{2} / E^{2}\right)$. This procedure absorbs in the iteration many non-dominant terms in the double emission exact matrix element (which we also calculated with REDUCE). The result is

$$
P\left(z, q_{\mathrm{T}}, s\right)=\frac{1}{1-z} \frac{1}{q_{\mathrm{T}}^{2}}\left[1+z^{2}-2 \frac{q_{\mathrm{T}}^{2}}{s}\left(1-z-\frac{q_{\mathrm{T}}^{2}}{s}\right)-z(1-z) \frac{2 M_{i}^{2}}{q_{\mathrm{T}}^{2}}\right],
$$

where new definitions are used ( $\beta$ is the velocity of the electron or positron)

$$
z=1-k, \quad q_{\mathrm{T}}^{2}=\left|M_{i+1}^{2}-M_{i}^{2}\right|, \quad\left|M_{2}^{2}-M_{1}^{2}\right|=E^{2} k(1-\beta \cos \theta) .
$$

The effect of eq. (20) is to modify the probability density in phase space. However, the final algorithm retains the physical property of evolution of the mass, as described in eq. (15). Eqs. (18) and (19) describe 3-momentum conservation but now must be used with $\left(p_{\mathrm{L}}, p_{\mathrm{T}}\right)$ as they can be extracted from $\left(z, q_{\mathrm{T}}\right)$ generated by eq. (20). Finally, the first-order $K$-factor [1]:

$$
K=1+(\alpha / \pi)\left(\frac{1}{3} \pi^{2}-\frac{1}{2}\right)
$$

is added as an overall normalization constant. This simple modification reproduces the exact next-to-leading result [1-3]. To summarize: This algorithm

(1) uses special kernels, which are equivalent to the exact first-order matrix element [eq. (20)];

(2) evolves independently the two legs and, on each leg, conserves 4-momentum [eq. (19)], evolves the mass [eq. (16)] and the cutoff [eq. (10)] in order to achieve factorization of the non-leading terms.

\section{Results and conclusions}

The comparison between an analytic calculation for the $Z^{0}$ shape [19] and the Monte Carlo is shown in fig. 4, with the real-virtual pairs terms removed [10]. The agreement between the two predictions is completely consistent with the statistical error ${ }^{\star}$ of the Monte Carlo. Fig. 5 shows the radiative corrections to the process $\mathrm{e}^{+} \mathrm{e}^{-} \rightarrow \nu \nu \gamma$. The solid line is from an analytic calculation [16] and the data points are from our Monte Carlo. Events are counted if they have only one photon with

* To our surprise, the disagreement between the fitting function and the Monte Carlo was nowhere worse than $0.3 \sigma$, where $\sigma \approx 0.3 \%$ near the peak and $\sigma \approx 1 \%$ on the tail. The difference arises from using the Monte Carlo in a weighted mode for this particular set of runs. Weighted and unweighted mode have been checked to be equivalent. 

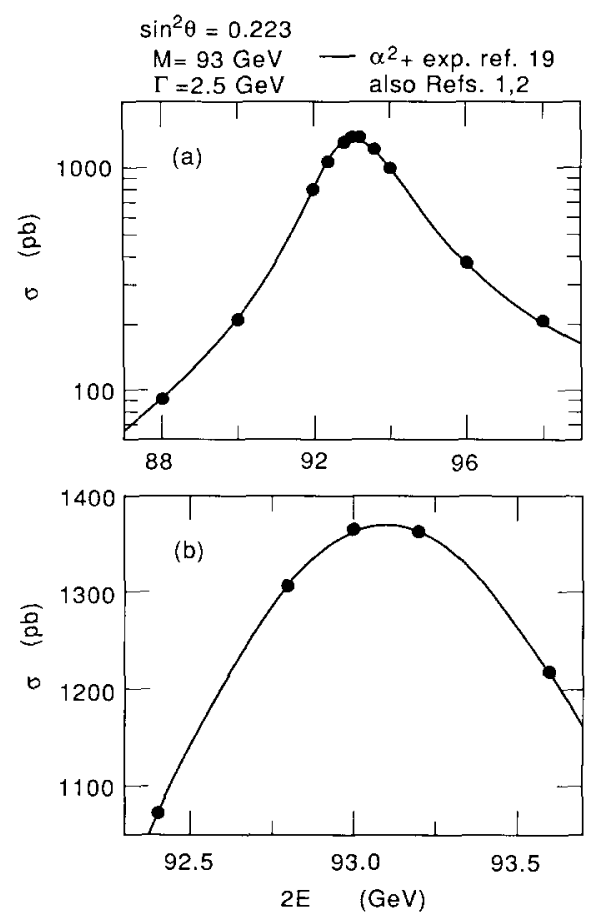

Fig. 4. Radiative corrections to the $\mathrm{Z}^{0}$ line shape in the $\mu \mu$ channel as predicted by the Monte Carlo (data points), and by an analytic exact $O\left(\alpha^{2}\right)$ exponentiated calculation from ref. [19]. According to ref. $[10]$, the predictions of refs. $[1,2,19]$ are indistinguishable. The $Z^{0}$ resonance parameters are: $M=93$ $\mathrm{GeV}, \Gamma(M)=2.5 \mathrm{GeV}$, and $\sin ^{2} \theta_{\mathrm{w}}=0.223$. The Monte Carlo was run with $10^{5}$ events per point, a cutoff $x_{0}$ of $10^{-5}$ and $s^{\prime}>0.1 s:$ (a) $87 \geq \sqrt{s} \leq 99 \mathrm{GeV}$; (b) $92.3 \geq \sqrt{s} \geq 93.7 \mathrm{GeV}$.

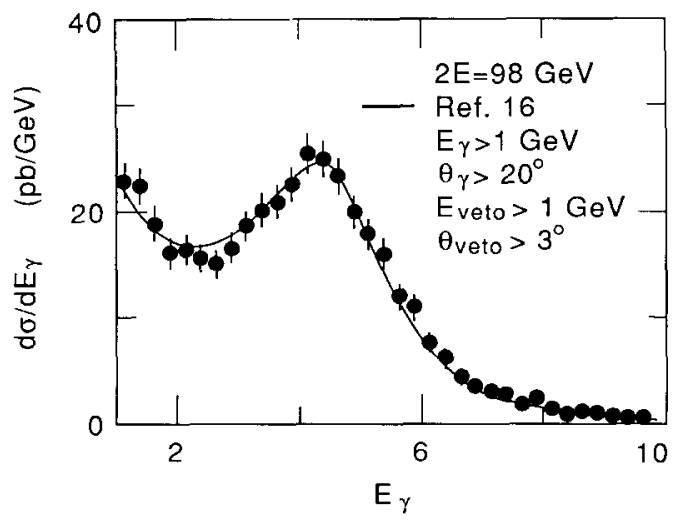

Fig. 5. Radiative corrections to $\gamma \nu \nu$ photon energy spectrum as predicted by the Monte Carlo (data points), and by an analytic exponentiated calculation from ref. [16]. The relevant parameters are: $M=93.2 \mathrm{GeV}, \Gamma(M)=2.6 \mathrm{GeV}, \sin ^{2} \theta_{\mathrm{w}}=0.223, \sqrt{s}=98 \mathrm{GeV}, x_{0}=10^{-5}$ and 3 species of neutrino. Photons are required to have an energy $E_{\gamma}>1 \mathrm{GeV}$, zenith $\theta_{\gamma}>20^{\circ}$ and are vetoed if another photon of energy greater than $1 \mathrm{GeV}$ has $\theta>3^{\circ}$. 
energy $E_{\gamma}>1 \mathrm{GeV}$, zenith $\theta_{\gamma}>20^{\circ}$ and no other photons above a $1 \mathrm{GeV}$ threshold and outside a $3^{\circ}$ cone around the beam pipe. Two comments are in order.

First, the slight disagreement in the $1-4 \mathrm{GeV}$ region $(\approx 5 \%)$ is due to the lack of logarithmic terms [see eq. (12)] in ref. [16], and to the independent emission modeling that does not reproduce the evolution of the mass and $p_{\mathrm{T}}$ [see eqs. (15) and (21)]. Both effects decrease the peak in fig. 5 less than $1 \%$, but are more significant in the broad valley on the left. The first effect is estimated to be $\approx-2 \%$ at $2 \mathrm{GeV}$. The second has been estimated by writing an independent emission (IE) Monte Carlo and by comparing it with the one described in the previous sections. The effect is $\approx-2 \%$. Our MC and the IE one differ significantly in the configuration described above only in what follows. Because our MC evolves $p_{\mathrm{T}}$ and mass, the unvetoed rate at large angle is $15 \%$ higher than in the IE case. However, the same effect increases the veto-ing probability of about the same amount, leaving approximately the same total rate but different veto-ing rates. On the peak no further hard photons are emitted and ref. [16] compares well with our algorithm. A comparison with $\mathrm{O}(\alpha)$ calculations would not be significant because the peak/valley ratio, which dominates the corrections to the shape of the spectrum, has $\mathrm{RC}$ of order $50 \%$. It is obvious that, near the Z-peak, the neutrino counting experiment cannot be corrected properly with a first-order calculation or an independent emission model.

Second, in ref. [16] the $O\left(\alpha^{2}\right)$ calculation is a one-photon $[O(\alpha)]$ cross section, radiatively corrected. On the contrary, in our Monte Carlo the evolution starts from the pure annihilation Born term. The agreement (with the limitations discussed above) between the two predictions is a direct consequence of the capability of the algorithm to achieve the next-to-leading result, and its capability to predict $\mathrm{RC}$ both inclusively and exclusively.

In fig. 6, we show the detailed shape of the initial state radiator [16] in the large energy loss region as compared again with an analytic calculation. In fig. 7 , the photon multiplicity for the initial state radiator is finally shown. When the radiator is effectively damped by the resonance, both the hard part of the photon spectrum and the high multiplicity tail are suppressed.

We conclude by discussing the physical content of our method. The methods generally used to calculate RC to the data [9], which are truncated expansions, always put a lower limit on the cutoff $[\bar{n}<1$, eq. (9)]. The optimum cutoff is then unrelated to the physical parameters of the experiment, and is neither the energy resolution, nor a bookkeeping parameter. Rather, the cutoff is an arbitrary external parameter which is tuned to optimize the approximation of the calculation and the results of the Monte Carlo are cutoff-dependent [9].

In the algorithm presented here, the cutoff can be set arbitrarily low [as introduced in eq. (8)]. The lower the cutoff, the more steps there will be in the evolution of the system. Results are independent of large variations of the cutoff (we have tried up to a minimum, computer-limited cutoff of $10^{-7}$ ), as long as the cutoff is 


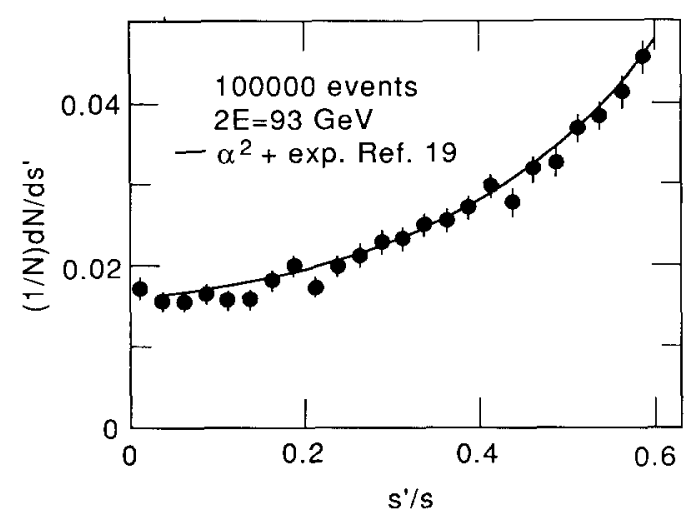

Fig. 6. The $s^{\prime}$ spectrum in the large energy loss region, as calculated by the Monte Carlo. The relevant parameters are the same as in fig. 4 and the spectrum is not weighted for the Born term.

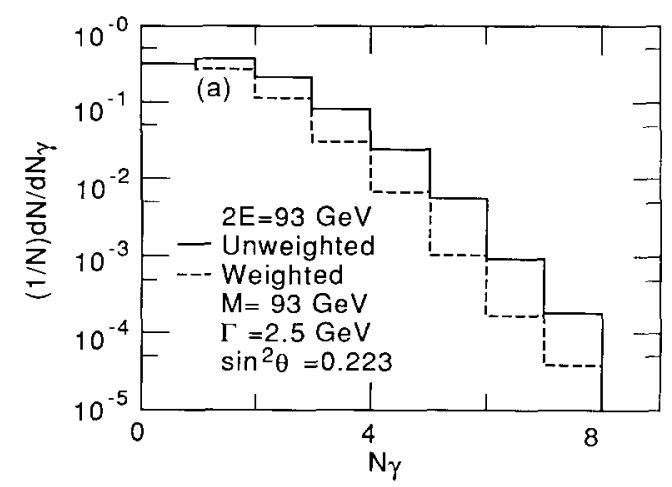

Fig. 7. Photon multiplicity as calculated by the Monte Carlo with the same parameters as in fig. 4. Solid line: the multiplicity unweighted by the Born term. Dashed line: the multiplicity weighted by the Born term.

kept much less than all the experimental scales. Operatively this means that the cutoff, to be physical, should be always much less than the minimum of the beam energy spread, acollinearity and one photon resolution of the experiment. In sects. 2 and 3 we have shown that the $\alpha^{2} L^{2}, \alpha^{2} L l$ and $\alpha^{2} l^{2}$ terms are correctly included in our recipe, as well as more dominant logarithms. Fig. 4 in the soft region and fig. 6 in the hard region empirically support our statement.

$\mathrm{Re}$-absorption of non-leading terms in the evolution of a few quantities (in our case the cutoff and the masses) has long been known to be a standard procedure in QCD [20]. In our case this is particularly simple due to the much simpler branchings 
and quantum numbers in QED. Further reasons for the success of this approach are the following advantages over QCD:

(1) The initial state [the Dirac $\delta$ in eq. (1)] is known exactly.

(2) The evolution is chain-like as opposed to tree-like (that is, one or two of the three particles at the elementary vertex are on mass-shell). This also has the advantage of eliminating sea electrons from the picture. Four-fermions amplitudes are small $(\approx 1 \%)$ and can be dealt with in other ways.

(3) The integral on $\mathrm{d} s^{\prime}$ [eq. (1)] is solvable exactly, with or without virtual pairs, therefore generating an exact statistics without loss of next-to-leading terms as in QCD.

As a last remark, chain-like evolution allows a predetermination of the number of steps of the evolution, and the evolution can start from the external relativistic invariant down to the inner line. Since it is the external relativistic invariant which enters in the exact matrix element [in the case of the initial state, see eqs. (20) and (8)], we have seen that one obtains a very natural inclusion of non-leading pieces in the evolution. Not surprisingly, this Monte Carlo evolves oppositely to well-known QCD examples [21] and, in the soft limit, explicitly calculates the Sudakov form factor [22].

We would like to thank M. Greco for constant encouragement and R. Frey for a careful reading of the manuscript. G.B. thanks S.J. Brodsky for many interesting discussions and L.T. thanks Stefano Catani and Oreste Nicrosini for many useful comments and discussions.

\section{Appendix}

We start from the following calculus theorem [23]:

$$
\frac{\mathrm{d}}{\mathrm{d} u} \int_{h(u)}^{g(u)} f(u, v) \mathrm{d} v=\frac{\mathrm{d} g}{\mathrm{~d} u} f[u, g(u)]-\frac{\mathrm{d} h}{\mathrm{~d} u} f[u, h(u)]+\int_{h(u)}^{g(u)} \frac{\partial}{\partial u} f(u, v) \mathrm{d} v
$$

Let us now consider the $\alpha^{2}$ term of the expansion [eq. (6)], which describes the emission of two real photons

$$
\int_{x / 1-x_{0}}^{1-x_{0}} P(z) P\left(\frac{x}{z}\right) \frac{\mathrm{d} z}{z}
$$

The upper limit is the separation between the no-emission region and the real emission region (i.e. the quantity called $B(0)$ in the text is the sum of all pieces of the expansion integrated between $1-x_{0}$ and 1). The lower limit corresponds, in the 
Feynman diagram modeling, to the separation between two real photons and one real photon plus one virtual photon. The integral function $Q(y)$ is defined as follows

$$
Q(y)=\int_{y}^{1-x_{0}} P\left(z^{\prime}\right) \mathrm{d} z^{\prime}, \quad Q\left(1-x_{0}\right)=0 .
$$

We have

$$
\begin{aligned}
\int_{x /\left(1-x_{0}\right)}^{1-x_{0}} P(z) P\left(\frac{x}{z}\right) \frac{\mathrm{d} z}{z} & =\int_{x /\left(1-x_{0}\right)}^{1-x_{0}} P(z) \frac{\partial}{\partial x} Q\left(\frac{x}{z}\right) \mathrm{d} z \\
& =\int_{x /\left(1-x_{0}\right)}^{1-x_{0}} \frac{\partial}{\partial x}\left[P(z) Q\left(\frac{x}{z}\right)\right] \mathrm{d} z
\end{aligned}
$$

and

$$
\begin{aligned}
\int_{x /\left(1-x_{0}\right)}^{1-x_{0}} \frac{\partial}{\partial x}\left[P(z) Q\left(\frac{x}{z}\right)\right] \mathrm{d} z=\frac{\mathrm{d}}{\mathrm{d} x} \int_{x /\left(1-x_{0}\right)}^{1-x_{0}} P(z) \mathrm{d} z \int_{x / z}^{1-x_{0}} P\left(z^{\prime}\right) \mathrm{d} z^{\prime} \\
-\frac{\mathrm{d}\left(1-x_{0}\right)}{\mathrm{d} x} P(z) Q\left(\frac{1-x_{0}}{z}\right)+\frac{\mathrm{d}\left(x /\left(1-x_{0}\right)\right)}{\mathrm{d} x} P(z) Q\left(1-x_{0}\right) .
\end{aligned}
$$

The second term in the right-hand side is zero because of the derivative of a constant, and the third term is zero because of $Q\left(1-x_{0}\right)$. The following identity holds

$$
\int_{x /\left(1-x_{0}\right)}^{1-x_{0}} P(z) P\left(\frac{x}{z}\right) \frac{\mathrm{d} z}{z}=\frac{\mathrm{d}}{\mathrm{d} x} \int_{x /\left(1-x_{0}\right)}^{1-x_{0}} \int_{x / z}^{1-x_{0}} P(z) P\left(z^{\prime}\right) \mathrm{d} z \mathrm{~d} z^{\prime},
$$

and the integration area in the $\left(z, z^{\prime}\right)$ space can be rewritten as

$$
z, z^{\prime}<1-x_{0}, \quad z z^{\prime}>x .
$$

\section{References}

[1] E.A. Kuraev and V.S. Fadin, SJNP 41(3) (1985) 466

[2] O. Nicrosini and L. Trentadue, Phys. Lett. B196 (1987) 551

[3] F.A. Berends, G.J.H. Burgers and W.L. Van Neerven, Nucl. Phys. B297 (1988) 429

[4] J.E. Campagne and R. Zitoun, LPNHEP-88-06

[5] G. Altarelli and G. Martinelli, CERN/EP 86-02, p. 47

[6] E. Etim, G. Pancheri and B. Touschek, Nuovo Cim. 51 (1967) 276

[7] M. Greco et al., Phys. Lett. B56 (1975) 367

[8] F. James, Rept. Prog. Phys. 43 (1980) 1145

[9] F.A. Berends and R. Kleiss, Nucl. Phys. B177 (1981) 237

[10] J. Alexander et al., PRD37: 56, 1988

[11] D. Kennedy et al., SLAC-PUB- 4128 
[12] J.E. Campagne and R. Zitoun, private communication

[13] F. Bloch and A. Nordsieck, Phys. Rep. 52 (1937) 54

[14] G. Bonneau and F. Martin, Nucl. Phys. B27 (1971) 381

[15] A. Bassetto, M. Ciafaloni and G. Marchesini, Nucl. Phys. B163 (1980) 477

[16] O. Nicrosini and L. Trentadue, Nucl. Phys. B318 (1989) 1

[17] F.A. Berends and R. Kleiss, Nucl. Phys. B260 (1985) 32

[18] A.C. Hearn, Rand Publication CP78

[19] F.A. Berends, G.J.H. Burgers and W.L. Van Neerven, Phys. Lett. B185 (1987) 395

[20] G. Marchesini and B.R. Webber, Nucl. Phys. B238 (1984) 1

[21] M. Bengtsson, T. Sjostrand and M. Van Zijl, ZPC 32: 67, 1986

[22] V.V. Sudakov, ZHETF 30 (1956) 187

[23] C.J. Everett and D.E. Cashwell, LA-9721-MS 